\title{
Erratum to: An overview of spectrum sensing techniques for cognitive LTE and LTE-A radio systems
}

\author{
Emad Hmood Salman ${ }^{1}$ - Nor Kamariah Noordin ${ }^{1,2}$ - Shaiful Jahari Hashim ${ }^{1}$. \\ Fazirulhisyam Hashim ${ }^{1}$. Chee Kyun $\mathrm{Ng}^{1,3}$
}

Published online: 27 September 2016

(C) Springer Science+Business Media New York 2016

\section{Erratum to: Telecommun Syst \\ DOI 10.1007/s11235-016-0221-z}

The first author's name was misspelled. "Emad Hamood Salman" should be "Emad Hmood Salman". The original article was corrected.

The online version of the original article can be found under doi:10.1007/s11235-016-0221-z.

$凶$ Chee Kyun Ng

mpnck@upm.edu.my

Emad Hmood Salman emad.h.salman@ieee.org

Nor Kamariah Noordin nknordin@upm.edu.my

Shaiful Jahari Hashim sjh@upm.edu.my

Fazirulhisyam Hashim

fazirul@upm.edu.my

1 Department of Computer and Communication Systems Engineering, Faculty of Engineering, Universiti Putra Malaysia, 43400 UPM Serdang, Selangor Darul Ehsan, Malaysia

2 Wireless and Photonics Network (WiPNeT) Research Centre, Universiti Putra Malaysia, 43400 UPM Serdang, Selangor Darul Ehsan, Malaysia

3 Malaysian Research Institute on Ageing, Universiti Putra Malaysia, 43400 UPM Serdang, Selangor Darul Ehsan, Malaysia 